\title{
Computer tomography guided transthoracic periaortic abscess needle biopsy in late mediastinitis after heart surgery
}

\author{
Tomasz Urbanowicz, Piotr Buczkowski, Wiktor Budniak, Izabela Katyńska, \\ Maciej Walczak, Jadwiga Tomczyk, Marek Jemielity \\ Department of Cardiac Surgery and Transplantology, Chair of Cardio-Thoracic Surgery, \\ Poznan University of Medical Sciences, Poznan, Poland
}

\begin{abstract}
Mediastinitis is a well-known complication of open heart surgery. Abscess as late complication, presenting years after heart surgery, is adegnotical. Transthoracic needle biopsies of lung parenchyma guided by computer tomography are widely accepted. The puncture of periaortic masses is not routinely performed. We report the case of an encapsulated mediastinal abscess localized next to ascending vascular graft. The febrile 47-year-old white male patient with history of Bentall operation was admitted to Cardiac Surgery Department. He was transferred for urgent chest tomography after International Normalization Ratio was reversed by prothrombin complex concentrate. Tomography revealed $7 \times 5 \times 4 \mathrm{~cm}$ mass between the sternum and ascending aorta, that was punctured by the needle. After biopsy specimen was sent for microbiology, the patient was transferred for surgery. There was no vascular graft invasion by the mass. The surgery was limited to abscess removal with postoperative drainage of periaortic area. The 6-week antibiotic therapy was applied. Patient recovered uneventfully. (Cardiol J 2014; 21, 3: 325-328)
\end{abstract}

Key words: late mediastinitis, heart surgery, Bentall surgery, periaortic abscess, computer tomography guided biopsy, transthoracic needle biopsy, prothrombin complex concentrate

\section{Introduction}

Mediastinitis is a well-known complication of open heart surgery. This situation is related to sternum infection and has been reported in less than $2 \%$ of procedures $[1,2]$. Mediastinal abscess as late complication, presenting years after the open heart surgery, is adegnotical [3]. Although transthoracic lung needle biopsy performed under computer tomography (CT) guidance is widely accepted, the periaortic abscess puncture has not been reported. We report the case of an encapsulated mediastinal abscess localized next to ascending aorta vascular graft. It was diagnosed nine years following the Bentall procedure, after patient became symptomatic, following lung infection. The abscess contents was obtained by puncture biopsy under CT guidance. It allowed not only proper diagnosis but was detrimental in decision making in referring patient for urgent surgery.

Address for correspondence: Tomasz Urbanowicz, MD, Department of Cardiac Surgery and Transplantology, Chair of Cardio-Thoracic Surgery, Poznan University of Medical Sciences, ul. Długa 1/2, 61-848 Poznań, Poland, tel: +48 6185492 10, fax: +48 6185490 85, e-mail: tk.urbanowicz@gmail.com 




Figure 1. Periaortic abscess next to ascending aorta in computer tomography.

\section{Case description}

We present a case of 47-year-old febrile white male patient admitted to the Cardiology Ward. He has given a 10-day history of persistent fever and chills. The patient's medical history was significant for Bentall procedure due to ascending aorta aneurysm 9 years earlier. He was on antibiotic therapy 4 weeks ago due to lung infection.

On admission, in laboratory findings white blood count was $18.3 \times 10^{9} / \mathrm{L}$, procalcitonin level was $10 \mathrm{ng} / \mathrm{mL}$ and C-reactive protein was $217 \mathrm{ng} / \mathrm{L}$. Blood pressure was $90 / 60 \mathrm{~mm} \mathrm{Hg}$ and heart rate was $110 \mathrm{bpm}$. Patient was treated with ciprofloxacinum for 10 days with no clinical response and blood samples were positive for Streptococcus aureus.

Patient was referred for urgent chest CT. On admission, the International Normalization Ratio (INR) was 3.2 and prothrombin complex (Prothromplex Total NF, Baxter) in the dose of $2,400 \mathrm{U}$ was given to reverse warfin action before tomography. One hour after Prothromplex Total NF admission, the INR was 1.2 and patient was sent for CT scan with diagnostic biopsy.

On CT irregular hypodense mass between the sternum and ascending aorta $7 \times 5 \times 4 \mathrm{~cm}$ was observed, as presented on Figure 1. Under CT guidance, the abscess was punctured and sample was obtained, as presented on Figure 2. The puncture was done through third right intercostal space, in the distance of $0.5 \mathrm{~cm}$ from sternum border, medially from right internal mammary artery. In CT scans, pulmonary densities in $6^{\text {th }}, 8^{\text {th }}, 9^{\text {th }}$ and $10^{\text {th }}$ right pleural segments were observed, too.

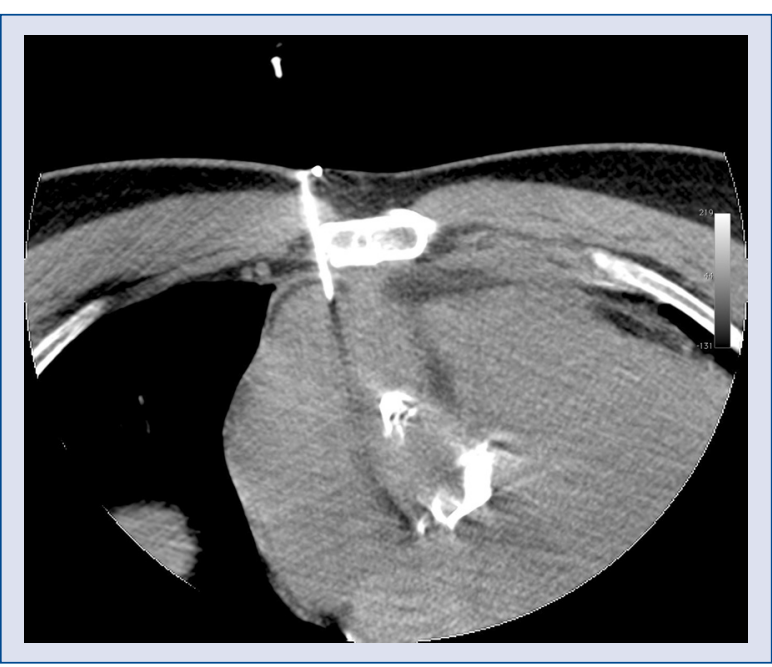

Figure 2. Periaortic needle biopsy guided by computer tomography scan.

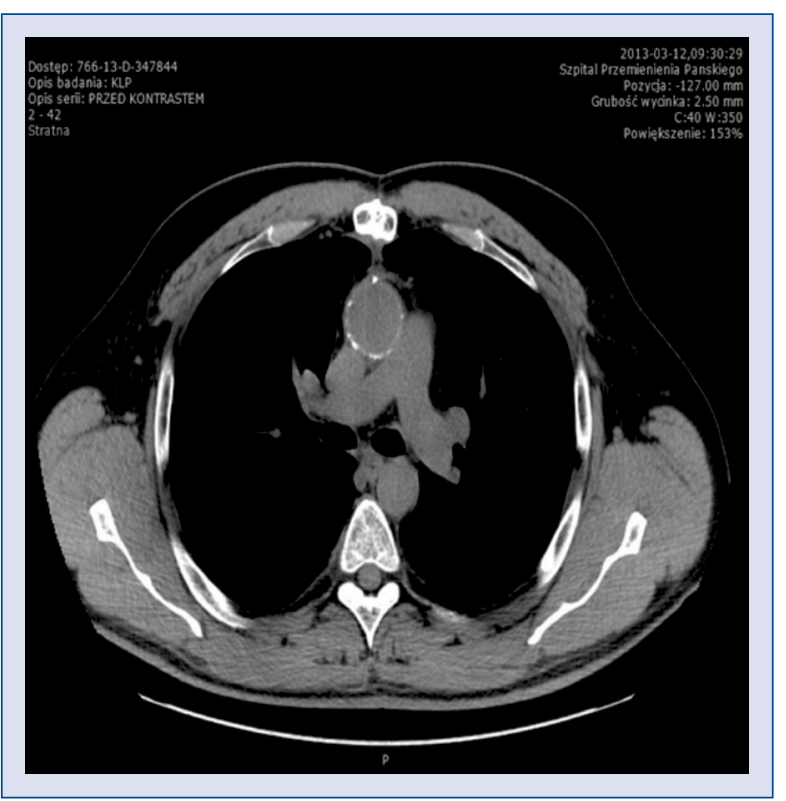

Figure 3. Resolution of periaortic mass on repeated computer tomography scan performed 6 weeks following surgery.

On the following day patient was transferred for operation. The procedure was performed through resternotomy, the abscess was excised. The mass was situated next to ascending aorta graft but not invading it. The procedure was limited to abscess excision and active drainage was applied for next 10 days. The antibiotic therapy (Vancomycin and Tazocin) was continued for 6 weeks. The CT scan was performed in the end of the antibiotic therapy, that revealed resolution in the side of the previously reported mass (Fig. 3). 
Patient was discharged home without any signs of infection. He remained in good condition for 6 months.

\section{Discussion}

The incidence of postoperative mediastinitis is reported in less than $2 \%$. There are hardly any reports of the incidence of late postoperative mediastinitis after cardiac surgery in the absence of sternal wound infection or dehiscence, especially following lung infection. Mediastinitis is usually early postoperative complication related to undergoing disease or intraoperative contamination. It is declared as systemic bacterial invasion combined with infected sternal dehiscence [4].

The unique situation of our patient is the presence of mediastinal abscess following lung infection that is located next to vascular graft of ascending aorta 9 years after the initial cardiac procedure. In literature, mediastinal abscess described as late as 5 years after coronary artery bypass grafting has been reported [5].

Staphylococcal infection, in contrary to streptococcal, is well known to predispose to the formation of encapsulated and localized infections. There are 2 possible scenarios of the origin of the abscess. A very low grade infection could stay dormant for a very long period of time. Another scenario is transient bacteremia giving rise to a metastatic abscess in an old hematoma. The most likely origin of mediastinal abscess formation in presented situation is the complication of lung infection.

Although there is no literature describing CT-guided thransthoracic periaortic punctures, needle biopsies are an accepted procedure for diagnosing lung lesions [6]. They can provide not only high diagnostic accuracy but have also relatively low complication rate in terms of intrapulmonary changes. The complications can include pneumothorax, bleeding, air embolisms, neoplastic seeding [7]. Periaortic biopsies are regarded as high risk procedures.

The abscess biopsy was performed under $\mathrm{CT}$ guidance. It allowed to obtain the specimen from the very proximity of the aortic graft. As the patient was admitted to hospital febrile, with symptoms of chills, generalized malaise, the diagnostic tomography was performed without any delay. The high INR ratio (3.2) was reversed by $2,400 \mathrm{U}$ of Prothromplex Total NF (Baxter). The control INR was performed 30 min after the infusion was completed, giving the normalized ratio (1.2).
There was $20 \mathrm{~mL}$ of puss obtained from the biopsy without any complications. It is worth to add that other pathologies that may present as anterior mediastinal masses postoperatively are mediastinal chylomas or ruptured pseudo aneurysms of aorta.

Although prothrombin complex concentrates (PCC) were originally developed for the treatment of bleeding disorders in patients with hemophilia B, their indications have shifted towards urgent reversal of over-anticoagulation with warfarin [8]. Vitamin $\mathrm{K}$ antagonists act through the inhibition of vitamin K-dependent gamma-carboxylation of coagulation factors II, VII, IX and X and also of the endogenous anticoagulation factors proteins $\mathrm{C}$ and S, synthesized in the liver [9]. It is difficult to predict an individual patient's response, vitamin $\mathrm{K}$ can be generally given unless the patient is actively bleeding, because of the longer time required to reverse the over-anticoagulation [9]. PCC have several advantages over fresh frozen plasma (FFP). First, various comparative studies have demonstrated that PCC are more effective than FFP at correcting INR [10, 11]. Furthermore, mean correction time was reported to be shorter with PCC than with FFP (41 vs. $115 \mathrm{~min}$ ) [12-14]. Another major advantage of PCC over FFP is that smaller volumes of the former are required to reverse anticoagulation. This is because the concentration of clotting factors in PCC is approximately 25 times higher than that in human plasma [15]. Another important consideration is the association of FFP with the risk of transfusion-related acute lung injury [16]. This risk is not present with the use of $\mathrm{PCC}$ as the antibodies responsible for this complication are removed during the process of manufacturing [17].

During surgery, the borders of abscess were localized next to the graft. There were no signs of graft infection so the vascular graft was not removed. The whole abscess cavity could not be removed as that would have mandated a complete ascending aortic replacement. The remnants of the cavity, next to the ascending aorta, were rasped very cautiously. The drainage of the abscess was constituted and the graft was left intact. There were two Redon drains left around the ascending aorta and for the following 8 days the antibiotics were given directly next to the graft. The sternum was healthy and was sought to be closed primarily.

\section{Conclusions}

Periaortic needle biopsy guided by CT scan can be performed safely giving proper diagnosis. 
Late periaortic mass can be successfully treated by partial excision compounded by 6 weeks antibiotic therapy.

\section{Conflict of interest: none declared}

\section{References}

1. Ottino G, De Paulis R, Pansini S et al. Major sternal wound infection after open heart surgery: A multivariate analysis of risk factors in 2579 consecutive operative procedures. Ann Thorac Surg, 1987; 44: 173.

2. Stable E, Tamunelin A, Bergstrom R et al. Sternal wound complications: Incidence, microbiology and risk factors. Eur J Cardiothorac Surg, 1997; 11: 1146.

3. Kaul P, Qadri SSA, Riaz M. Chronic encapsulated mediastinal abscess presenting with remote cutaneous fistulization 12 years after redo aortic valve replacement for prosthetic valve endocarditis. J Cardiothorac Surg, 2006; 1: 22.

4. Nikol S, Huchns TY, Pilz G, von-Schidt W. Conservative treatment of a paracardial abscess following post infarct patching. Scand Cardiovasc J, 1998; 32: 49-50.

5. Domaradzki W, Olszowka P, Mrozek R et al. Mediastinal abscess as a remote complication of CABG combined with temporary cardiac pacing. Kardiol Pol, 2005; 63: 70-72.

6. Ohno Y, Hatabu H, Takenaka D et al. CT-guided transthoracic needle aspiration biopsy of small $(\leq 20 \mathrm{~mm})$ solitary pulmonary nodules. AJR, 2003; 180: 1665-1669.

7. Lopez Hanninen E, Vogl TJ, Ricke J, Felix R. CT-guided percutaneous core biopsies of pulmonary lesions: diagnostic accuracy, complications and therapeutic impact. Acta Radiol, 2001; 42: 151-155.
8. Hellstern P, Halbmayer WM, Kohler M et al. Prothrombin complex concentrates: indications, contraindications, and risks: A task force summary. Thromb Res, 1999; 95: S3-S6.

9. Ansell J, Hirsh J, Poller L et al. The pharmacology and management of the vitamin K antagonists. The Seventh ACCP Conference on Antithrombotic and Thrombolytic Therapy. Chest, 2004; 126 (suppl. 3): 204S-233S.

10. Erber WN, Perry DJ. Plasma and plasma products in the treatment of massive haemorrhage. Best Pract Res Clin Haematol, 2006; 19: 97-112.

11. Makris M, Greaves M, Phillips WS et al. Emergency oral anticoagulant reversal: the relative efficacy of infusions of fresh frozen plasma and clotting factor concentrate on correction of the coagulopathy. Thromb Haemost, 1997; 77: 477-480.

12. Cartmill M, Dolan G, Byrne JL, Byrne PO. Prothrombin complex concentrate for oral anticoagulant reversal in neurosurgical emergencies. Br J Neurosurg, 2000; 14: 458-461.

13. Imberti D, Barillari G, Biasioli $C$ et al. Prothrombin complex concentrates for urgent anticoagulation reversal in patients with intracranial hemorrhage. Pathophysiol Haemost Thromb, 2009; 36: 259-265.

14. Leissinger CA, Blatt PM, Hoots WK, Ewenstein B. Role of prothrombin complex concentrates in reversing warfarin anticoagulation: A review of the literature. Am J Hematol, 2008; 83: 137-143.

15. Vigué B, Ract C, Tremey B et al. Ultra-rapid management of oral anticoagulant therapy-related surgical intracranial hemorrhage. Intensive Care Med, 2007; 33: 721-725.

16. Bux J. Transfusion-related acute lung injury (TRALI): A serious adverse event of blood transfusion. Vox Sang, 2005; 89: 1-10.

17. Levy JH, Tanaka KA, Dietrich W. Perioperative hemostatic management of patients treated with vitamin $\mathrm{K}$ antagonists. Anesthesiology, 2008; 109: 918-926. 